\title{
Alter
}

Revue de phénoménologie

28 | 2020

La religion

\section{Lettre de Husserl à Przywara du 15 juillet 1932}

Traducteur : Dominique Pradelle

\section{CpenEdition}

Journals

Édition électronique

URL : https://journals.openedition.org/alter/2215

DOI : 10.4000/alter.2215

ISSN : 2558-7927

Éditeur :

Association ALTER, Archives Husserl (CNRS-UMR 8547)

Édition imprimée

Date de publication : 1 novembre 2020

Pagination : 317-320

ISBN : 978-2-9550449-6-4

ISSN : $1249-8947$

\section{Référence électronique}

«Lettre de Husserl à Przywara du 15 juillet 1932 », Alter [En ligne], 28 | 2020, mis en ligne le 22

décembre 2020, consulté le 12 décembre 2021. URL : http://journals.openedition.org/alter/2215 DOI : https://doi.org/10.4000/alter.2215

Ce document a été généré automatiquement le 12 décembre 2021.

Revue Alter 


\section{Lettre de Husserl à Przywara du 15 juillet 1932 \\ Traduction : Dominique Pradelle}

1 Nous présentons ci-dessous la traduction de : Husserl, Brief an Przywara, 15. VII. 1932, parue dans le Briefwechsel, Bd. VII: Wissenschaftlerkorrespondenz (éd. par E. et K. Schuhmann), Dordrecht, Kluwer Academic Publishers, 1994, p. 237-238.

2 Né le 12 octobre 1889 à Kattowitz et mort le 28 septembre 1972 à Hagen, Erich Przywara était un philosophe et théologien catholique allemand de la compagnie de Jésus. Son ouvrage le plus connu est précisément celui qu'il adresse à Husserl et qui fournit l'occasion de cette succincte correspondance: Analogia entis. Metaphysik, I : Prinzip, Munich, 1932 (trad. par Philibert Secretan, Analogia entis, Paris, Puf, 1990). L'analogie métaphysique (ou analogie de l'être, mais littéralement analogie de l'étant) est de provenance aristotélico-thomiste et se situe originellement dans le domaine de l'ontologie, c'est-à-dire de la connaissance de l'étant en tant que tel : en effet, si l'être de l'étant se dit de multiple façon ou admet une pluralité de sens, se pose alors le problème de l'unité de la doctrine de l'être, c'est-à-dire du rapport de ces diverses acceptions de l'être à un sens premier qui en constituerait un foyer de sens idéal - d'où l'égalité des rapports de ces différents sens au sens premier.

3 Ce motif de l'analogie se laisse ensuite transposer à la théologie, à savoir au rapport entre le sensible et le divin, et plus spécifiquement au rapport de l'homme à Dieu : si l'analogie est une égalité de rapports entre réalités d'ordre distinct, elle permet en effet de penser la similitude entre l'être de l'homme et celui de Dieu, tout autant que la manière qu'a l'homme de se rapporter à Dieu'1. C'est dans ce contexte théologique que Przywara s'oppose à la lecture que fait Karl Barth de l'Épitre aux Romains, qu'il juge comme étant l'affirmation d'une vision du monde spécifiquement protestante : celle d'un Dieu «tout autre", tout autre que tout étant non divin, donc excluant toute analogie avec quelque autre étant que ce soit, notamment l'homme. À cela, Przywara tâche d'opposer une Weltanschauung catholique, fondée sur l'analogie entre l'être de Dieu et de l'homme, nature et surnature, le visible et le fond caché, le Dieu intérieur qui requiert notre assentiment personnel et le Dieu supérieur et incompréhensible qui se manifeste dans la Création'. Ce qui est en jeu dans l'analogie comme principe d'une 
métaphysique, c'est donc la possibilité, d'une part, de faire l'expérience de la «totalité de l'ordre créé » et, d'autre part, de s'élever à un concept de Dieu qui demeure cependant immanent à la métaphysique.

Quel est le point de jonction entre ces considérations et la phénoménologie, qui pouvait pousser le père Przywara à adresser son ouvrage à Husserl ? C'est que, d'une manière qui évoque les deux versants husserliens de l'a priori de corrélation entre les types d'étant et leur mode de constitution par la conscience pure, Przywara thématise la double face de la métaphysique : la méta-ontique (métaphysique de l'étant) et la métanoétique (métaphysique de la conscience pure), avant de montrer que toute thématisation unilatérale et exclusive de l'une ou de l'autre conduit à des contradictions, et qu'il faut par conséquent prendre en vue la tension entre ces deux pôles en soi insaturés - tension qui ne se résout en unité qu'en Dieu. Ainsi se définit une métaphysique finie ou "créaturelle ", dont la tâche est de penser la tension et l'unité dynamiques entre être et conscience, qui font signe vers le concept d'un Dieu immanent à la métaphysique en lequel se résoudrait cette tension.

5 Cette transition de la corrélation entre la conscience et l'étant à un Dieu créateur permet à Przywara de réintroduire un motif thomiste, pour opposer les perspectives propres à la philosophie et à la théologie : dans la première Dieu est mesuré par la créature (c'est-à-dire élucidé à partir du sens que possède Dieu pour la conscience finie), tandis que dans la seconde la créature est mesurée par Dieu et en est la manifestation. C'est précisément ce saut vers la théologie que pointe Husserl lorsqu'il oppose ici, à la philosophie théologique de Przywara, sa propre «manière athéologique de philosopher » (atheologisches Philosophieren). La lettre désigne ainsi en creux, au-delà de cet athéologisme méthodique, l'exigence méthodique de toute phénoménologie de la religion et $d u$ divin: faire retour aux "évidences religieuses" (religiöse Evidenzen) qui sont donatrices du sens d'être de Dieu et qui en attestent la validité ontologique; car c'est uniquement à partir du mode d'accès de la conscience pure - qui n'est ni finie ni infinie, mais située en-deçà du partage entre le fini et l'infini - au divin et à Dieu que l'on peut élucider le sens d'être de Dieu et son mode d'attestation pour la conscience. C'est seulement ainsi que l'on peut parvenir à l'élucidation du «sens phénoménologique du mot "Dieu" ». Il reste à savoir si ce sens phénoménologique s'est déposé dans une religion particulière - est-ce le protestantisme, auquel Husserl avoue s'être tôt converti, saisi qu'il avait été par la lecture des Évangiles ? -, ou s'il se situe endeçà de la différence entre les religions, dans quelque théologie naturelle qui serait immanente à la conscience de transcendance divine.

[237]

Freiburg, le 15 VII. 1932 (copie)

Très digne et estimé père Przywara,

C'est de tout cœur que je vous adresse mes remerciements pour m'avoir gracieusement envoyé le tome premier de votre Métaphysique ${ }^{3}$. En toute sincérité, je dois vous avouer que c'est avec une envie confinant au péché que je m'en suis saisi et que je l'ai parcouru en diagonale - ce que je vous envie, c'est en effet qu'il vous soit donné d'être déjà parvenu à l'achèvement systématique d'un travail de pensée qui s'est étalé sur de longues années. Le fait de vous envier ne m'empêche cependant nullement de vous féliciter de tout cœur; cela va d'ailleurs de soi, vu le grand intérêt que m'inspirent le sérieux et l'élévation de vos efforts philosophiques, ainsi que l'intérêt philosophique que suscite en moi le parallèle entre votre manière théologique et ma manière 
athéologique de philosopher [Parallelität Ihres theologischen und meines atheologischen Philosophierens]. En ce qui me concerne, je suis encore en chemin mais, Dieu soit loué, je ne manque pas de force productrice et me consacre en ce moment à une purification ultime de la hiérarchie des niveaux [Stufenfolge] de la temporalité en lesquels s'expose, de façon systématique et vue de l'intérieur, la hiérarchie des niveaux de l'étant-qui-estpour-nous [Stufenfolge des für-uns-Seienden]. Qu'en suivant ce chemin de la constitution transcendantale, j'aie la certitude de parvenir en dernière instance à un achèvement " téléologique ", c'est là une chose que vous savez; vous savez aussi que je vis pénétré de la ferme conviction que ma méthode phénoménologique suffit à résoudre tous les véritables problèmes d'évidence [Evidenzproblemen], mais également qu'en effectuant une clarification réflexive des effectuations constitutives qui s'y sont déposées [in ihnen niedergeschlagene konstitutive Leistungen], elle permet d'accéder à l'élucidation ultime de la teneur de sens et de la portée de ces évidences. Cela vaut aussi pour les évidences religieuses [religiöse Evidenzen].

Voilà qui aidera les théologiens, même si de prime abord il leur semblera qu'il en découle de graves hérésies. Quant à m'appuyer sur le théisme de la tradition scolastique - entendue selon les explicitations usuelles qu'on donne de son sens -, voilà une chose que je refuse résolument. Bien entendu, je dis cela contre M. Keilbach ${ }^{4}$ et ses « preuves [de l'existence] de Dieu », dont il prétend que je les espérais (tout cela d'après des propos que j'aurais soi-disant tenus lors de conversations). Manifestement l'Idée, méthodiquement construite, d'un progrès de l'intelligence humaine s'élevant vers l'intelligence absolument illimitée, n'est pas entendue [238] comme une anticipation du sens phénoménologique véritable du mot «Dieu», mais seulement comme une Idée méthodique.

11 Que de votre côté vous soyez prémuni contre des choses si primitives, ce n'est pas seulement à la lecture de votre œuvre que j'en ai acquis la certitude. Certes, par son élaboration philosophico-théologique des nouveaux motifs phénoménologiques, cette œuvre suscitera un malaise chez ceux qui trouvent plus commode de modifier les formules traditionnelles que de s'ouvrir à ce que de leur temps on a pensé par soimême, comme l'ont fait les grands créateurs de la tradition en tant qu'ils ont pensé par eux-mêmes.

12 Je vous adresse à nouveau mes vœux sincères pour que votre nouveau tome parvienne à un accomplissement satisfaisant, et que votre œuvre tout entière ait un effet des plus propices.

\section{NOTES}

1. J.-F. Courtine, Inventio analogiae : Métaphysique et ontothéologie, Paris, Vrin, 2005.

2. Cf. J. Greisch, "Analogia entis et analogia fidei: une controverse théologique et ses enjeux philosophiques (Karl Barth et Erich Przywara) ", Les Etudes Philosophiques, mars 1989, p. 475-496.

3. E. Przywara, Analogia entis. Metaphysik, I : Prinzip, Munich, 1932 (le volume se trouve dans la bibliothèque de Husserl). 
4. En 1935, Wilhelm Keilbach reçut le grade de docteur à la Gregoriana de Rome, avec un travail intitulé Die philosophische Problematik der Religionen. Eine religionsphilosophische Studie mit besonderer Berücksichtigung der neuen Religionspsychologie [La problématique philosophique des religions. Une étude de philosophie de la religion, avec une prise en compte particulière de la psychologie de la religion]. 